\title{
Metales pesados en el ambiente: síndrome de Guillain-Barré like
} Heavy metals in the enviroment: Guillain-Barré like syndrome

\author{
Dra. Graciela M. Gaiolia, Bioq. Daniel E. González $z^{b}$ Bioq. Giselle Areny ${ }^{b}$, Dr. Marcelo Grela $y$ \\ Dr. Diego Amoedo ${ }^{a}$
}

\section{RESUMEN}

El síndrome de Guillain-Barré constituye una entidad de etiología diversa, que se caracteriza por debilidad muscular aguda, simétrica, ascendente y progresiva, y es una de las polineuropatías adquiridas más frecuentes en la infancia. Entre los diagnósticos diferenciales, deben considerarse las neuropatías producidas por metales pesados, mercurioy plomo, y metaloides, como el arsénico, plaguicidas organofosforados y el tetracloruro de carbono.

Se presenta a un paciente de 14 años con diagnóstico de síndrome de Guillain-Barré sin respuesta al tratamiento convencional con gammaglobulina. Considerando otras etiologías, se sospechó neuropatía producida por metales pesados, y se confirmó intoxicación por mercurio.

El objetivo de esta presentación es concientizar a los pediatras acerca del impacto de los tóxicos ambientales en la salud infantil para realizar un diagnóstico precoz pesquisando datos clave a través de la historia clínica ambiental.

Palabras clave: polineuropatías, intoxicación del sistema nervioso por mercurio, historia clínica ambiental.

\begin{abstract}
Guillain-Barré syndrome is an entity of diverse etiology, characterized by acute, symmetric, ascending and progressive muscle weakness, being one of the most frequent acquired polyneuropathies in childhood. Neuropathies produced by heavy metals, mercury and lead, and metalloids, such as arsenic, organophosphorus pesticides and carbon tetrachloride, should be considered among the differential diagnoses.

We present a 14-year-old patient with a presumptive diagnosis of Guillain-Barré syndrome without response to conventional treatment with gamma globulin. Considering other etiologies, heavy metal neuropathy was suspected, and mercury poisoning was confirmed.
\end{abstract}

a. Clínica ambulatoria.

b. Laboratorio de Toxicología.

Hospital de Pediatría S. A. M. I. C.

"Prof. Dr. Juan P. Garrahan".

c. Médico especialista en Medicina Familiar y Comunitaria del Hospital Provincial Dr. Fernando Irastorza de Curuzú Cuatiá, Corrientes.

Correspondencia:

Dra. Graciela M. Gaioli: mgaioli@gmail.com

Financiamiento: Ninguno.

Conflicto de intereses: Ninguno que declarar.

Recibido: 22-1-2019

Aceptado: 10-8-2019
The aim of this presentation is to make pediatricians aware about the impact of environmental toxic agents on children's health in order to make an early diagnosis by researching key data through the environmental clinical history.

Key words: polyneuropathies, mercury poisoning, nervous system, environmental clinical history.

http: / / dx.doi.org/10.5546/ aap.2020.e48

Cómo citar: Gaioli GM, González DE, Areny G, Grela M, Amoedo D. Metales pesados en el ambiente: síndrome de Guillain-Barré like. Arch Argent Pediatr 2020;118(1):e48-e52.

\section{INTRODUCCIÓN}

El mercurio $(\mathrm{Hg})$ es un contaminante ambiental de relevancia mundial por el impacto que genera en el neurodesarrollo y por las manifestaciones neurológicas y psiquiátricas que se pueden presentar en cualquier etapa de la vida. También afecta el sistema endócrino e inmune, el aparato digestivo, respiratorio y renal, y causa daños que pueden ser irreversibles. Atraviesa la placenta y pasa a la leche materna por su gran liposolubilidad. Una de las fuentes más significativas de exposición para el humano es a través de la dieta con pescados contaminados con metilmercurio (Me- $\mathrm{Hg})$, la especie orgánica de este metal pesado. ${ }^{1-5}$

\section{CASO CLÍNICO}

Paciente de sexo masculino de 14 años de edad, oriundo de la provincia de Corrientes, Argentina, previamente sano, que fue derivado al Hospital con sospecha de síndrome de GuillainBarré. Había comenzado 4 meses antes de la consulta con dolor lumbar y temblores. A las dos semanas de haberse iniciado los síntomas, el niño presentó debilidad de los miembros inferiores, sudoración profusa, exantema maculopapular con predominio en el tronco, eritema doloroso y lesiones queratodérmicas en las palmas y las plantas, que se descamaban rápidamente, temblor intencional, labilidad emocional, alteraciones del sueño y de la conducta. La madre refería el descenso de $7 \mathrm{~kg}$ de peso desde el inicio de los síntomas. No presentaba antecedentes de infección viral previa ni vacunas recientes. 
En el hospital de su lugar de origen, se habían realizado hemograma, hepatograma, uremia y creatininemia, que fueron normales, y examen de orina, con microhematuria. El estudio de líquido cefalorraquídeo (LCR) mostró una disociación albuminocitológica (recuento de células: 31 elementos mononucleares; glucorraquia de $88 \mathrm{~m} / \mathrm{dl}$; proteínas de $139 \mathrm{mg} / \mathrm{dl}$ ). La tomografía axial computada (TAC) cerebral fue normal. En la resonancia magnética nuclear $(\mathrm{RMN})$ de la columna y la médula, no se observaron lesiones óseas, pero sí un reforzamiento lineal y continuo de las raíces anteriores de la cola de caballo. El electromiograma presentó signos compatibles con radiculopatía primariamente desmielinizante de grado moderado.

Con el diagnóstico de síndrome de GuillainBarré, asociando la clínica del paciente, el estudio citoquímico del LCR, el electromiograma y la RMN compatibles, se indicó el tratamiento con gammaglobulina endovenosa. El paciente no presentó mejoría clínica, por lo cual fue derivado a nuestro Hospital para reevaluar el diagnóstico.

Se consideró el diagnóstico de neuropatía periférica asociado a intoxicación por metales pesados (que se había sospechado también en el hospital de origen) u otros tóxicos ambientales. Se solicitaron plombemia, cuantificación de mercurio $(\mathrm{Hg})$ y arsénico (As) en orina de $24 \mathrm{~h}$. La plombemia fue menor de $5 \mu \mathrm{g} / \mathrm{dl}$; el As urinario, de $6 \mu \mathrm{g} / \mathrm{l}$, y el $\mathrm{Hg}$ urinario, elevado, con un valor de 48,4 $\mu \mathrm{g} / 1$ (Tabla 1). En su domicilio, se realizó el análisis del agua de red y de la bajada del tanque, los cuales resultaron negativos.

Se consideró el cuadro como polineuropatía secundaria a intoxicación por mercurio. Se inició el tratamiento quelante con D-penicilamina a razón de $30 \mathrm{mg} / \mathrm{kg} /$ día durante 10 días en 3 ciclos, asociado a piridoxina, ya que la droga aumentaba los requerimientos de esta vitamina. Era un quelante de metales pesados que se utilizaba en tercera línea luego del etilendiaminotetraacético o succímero, que promovía su excreción por la orina. Es necesario el control de la función renal, hepática y hematológica mientras se prolongue la administración. ${ }^{6}$
Tras el primer ciclo, el paciente presentó una mejoría clínica progresiva tanto neurológica como psiquiátrica y, a los dos meses de haberse finalizado el tratamiento, logró la recuperación total del estado de salud (Tabla 2). Se indagó la fuente de exposición al Hg a través de la historia clínica ambiental y, luego de interrogarlo repetidas veces, el paciente relató que molía tubos fluorescentes en el patio de su casa a modo de "changa" para disminuir el volumen de residuos.

Se cuantificó $\mathrm{Hg}$ en la orina de una muestra aislada a sus 4 hermanos convivientes y a su padre, quienes se encontraban asintomáticos y no requirieron quelación. Conjuntamente con el Servicio Social de la zona, se eliminó la fuente de mercurio, fundamental para lograr la recuperación del paciente. Se le realizaron controles mensuales hasta obtener determinaciones de $\mathrm{Hg}$ urinario por debajo del valor de referencia.

\section{DISCUSIÓN}

El síndrome de Guillain-Barré es una de las polineurorradiculopatías adquiridas más frecuentes en la infancia. Se presenta con debilidad de los miembros, generalmente, simétrica, y pérdida progresiva de los reflejos hasta llegar a la arreflexia.

Los síntomas progresan en forma ascendente en menos de 4 semanas. Es característica la disociación albuminocitológica en el LCR a los 7-10 días de haberse iniciado el cuadro. El electromiograma muestra signos de desmielinización. ${ }^{1}$

Entre sus diagnósticos diferenciales, deben considerarse aquellos cuadros producidos por

TABLA 1. Valores de referencia de cuantificación de metales pesados

\begin{tabular}{lc}
\hline Cuantificación & Valores de referencia \\
\hline Plombemia & $<5 \mu \mathrm{g} / \mathrm{dl}$ \\
Arsénico urinario de $24 \mathrm{~h}$ & $<44,6 \mu \mathrm{g} / 1$ \\
Mercurio urinario de $24 \mathrm{~h}$ & $<14 \mu \mathrm{g} / 1$ \\
\hline
\end{tabular}

TABLA 2. Criterios de intervención según los valores detectados de mercurio en orina de $24 \mathrm{~h}$

\begin{tabular}{lcc}
\hline Nivel & Valor urinario & Intervención \\
\hline Bajo & Menor de $20 \mu \mathrm{g} / 1$ & Detectar la fuente y eliminar la exposición. \\
Medio & De 20 a $50 \mu \mathrm{g} / 1$ & Repetir la cuantificación. Evaluar la quelación de acuerdo con la clínica. \\
Elevado & Mayor de $50 \mu \mathrm{g} / 1$ & Repetir la cuantificación. De persistir elevado, tratamiento quelante. \\
\hline
\end{tabular}


metales pesados, como mercurio $(\mathrm{Hg})$ y plomo $(\mathrm{Pb})$, y también por metaloides, como el arsénico (As), plaguicidas y tetracloruro de carbono ${ }^{7}$ (Tabla 3).

El Hg es un importante tóxico ambiental con gran impacto sobre la salud humana, principalmente, por sus comprobados efectos neurotóxicos. Es un metal pesado, plateado, ubicuo; único líquido a temperatura ambiente que tiene la capacidad de formar amalgamas con otros metales y, en su forma elemental, se volatiliza fácilmente formando vapores incoloros e inodoros. ${ }^{2-5}$

Las fuentes naturales de Hg están representadas por las liberaciones provenientes de la movilización natural, tal como se encuentra en la corteza terrestre proveniente de la actividad volcánica, de la erosión de rocas o de los incendios forestales. El Hg se encuentra en el suelo, en el aire y en el agua, y se incorpora, de esta forma, a los ciclos biológicos y pasa a la atmósfera. En el agua y en el suelo, las bacterias metanógenas, a través de un proceso de metilación, transforman el $\mathrm{Hg}$ inorgánico en $\mathrm{Me}-\mathrm{Hg}$, la forma más tóxica de este metal para los seres vivos. Esta transformación lo hace más lipofílico, por lo cual se acumula fácilmente en la cadena trófica animal y se inicia el proceso de biomagnificación y bioacumulación en los animales superiores, sobre todo, en los peces, importante fuente de $\mathrm{Hg}$, y así la dieta pasa a ser la principal fuente de exposición para el hombre. ${ }^{8}$

El porcentaje de emisión de fuentes antropogénicas se duplicó en los últimos 100 años; estas son las responsables del $85 \%$ de las emisiones totales. Existen numerosas y variadas fuentes de exposición al mercurio (Tabla 4).

Para el diagnóstico, es importante realizar una historia clínica ambiental basándose en una anamnesis detallada y reiterada, en un examen físico minucioso, con estudios complementarios no específicos (hemograma, función hepatorrenal, electrocardiograma, electromiograma, punción lumbar) y específicos (determinación de mercurio en orina).

TABLA 3. Clasificación de los trastornos neuromusculares (modificado de Avaria et al.)

\begin{tabular}{|c|c|c|}
\hline Componente de la unidad motora afectado & Etiologías & Trastornos más frecuentes \\
\hline Motoneurona & $\begin{array}{l}\text { Hereditarias } \\
\text { Adquiridas }\end{array}$ & $\begin{array}{ll}\text { - } & \text { AME. } \\
\text { - } & \text { Virales: poliomielitis. } \\
\text { - } & \text { Degenerativas: ELA. } \\
\end{array}$ \\
\hline Nervio periférico & Hereditarias & $\begin{array}{l}\text { - } \text { Charcot-Marie-Tooth. } \\
\text { - Enfermedades neurodegenerativas: } \\
\text { leucodistrofias, ataxia telangiectasia, etc. } \\
\text { - Inflamatorias: Guillain-Barré, PCDI. } \\
\text { - Infecciosas. } \\
\text { - Tóxicas. } \\
\text { - Metabólicas: diabetes. }\end{array}$ \\
\hline Unión neuromuscular & $\begin{array}{l}\text { Hereditarias } \\
\text { Adquiridas }\end{array}$ & $\begin{array}{l}\text { - } \quad \text { Síndromes miasténicos congénitos. } \\
\text { - } \quad \text { Botulismo infantil. } \\
\text { - } \quad \text { Miastenia gravis. }\end{array}$ \\
\hline Músculo & Hereditarias & $\begin{array}{l}\text { - } \text { Distrofias musculares. } \\
\text { - Miopatías congénitas. } \\
\text { - Miopatías metabólicas. } \\
\text { - Glucogenosis. } \\
\text { - Mitocondriales. } \\
\text { - Trastornos de los canales iónicos. } \\
\text { - Inflamatorias. } \\
\text { - Tóxicas. } \\
\text { - Endócrinas. }\end{array}$ \\
\hline
\end{tabular}

AME: atrofias musculares espinales; ELA: esclerosis lateral amiotrófica;

PCDI: polineuropatía desmielinizante crónica inflamatoria. 
En la exposición crónica al $\mathrm{Hg}$, tanto inorgánico como orgánico, el sistema nervioso es el órgano blanco. La exposición crónica al Hg inorgánico compromete el sistema nervioso central y también se manifiesta como una neuropatía periférica por efecto directo del $\mathrm{Hg}$ sobre las terminaciones anteriores de las motoneuronas con degeneración axonal.

La tríada característica es el temblor en reposo o intencional, la gingivitis y el eretismo (cuadro psiquiátrico caracterizado por depresión, irritabilidad persistente, labilidad emocional, trastornos de la memoria, insomnio, demencia). ${ }^{8-13}$ Es frecuente la hipertensión arterial y la taquicardia, que incluye la taquicardia paroxística supraventricular y la miocardiopatía.

Las lesiones dermatológicas se caracterizan por eritema de las palmas y las plantas, luego descamación, hiperhidrosis, prurito y dolor, asociado a un rash inespecífico de localización proteiforme. En la infancia, se conoce como acrodinia y se encuentra relacionada con reacciones de hipersensibilidad al Hg. También puede observarse alopecia.

Esta intoxicación puede producir alteraciones inmunológicas con disminución de las poblaciones linfocitarias y aumento de los niveles de inmunoglobulina E, inhibir la síntesis de corticoides y alterar la producción de interferón e interleuquinas 2. El $\mathrm{Hg}$ se comporta como un disruptor endócrino que puede generar alteración de la función tiroidea y en la síntesis de hormona folículoestimulante (follicle stimulating hormone; $F S H$, por sus siglas en inglés), hormona luteinizante (luteinizing hormone; $L H$, por sus siglas en inglés), progesterona y andrógenos, y disminuye el tamaño testicular y produce disfunción eréctil. La frecuencia de abortos se encuentra aumentada frente a la exposición crónica. ${ }^{8-13}$

En cuanto al Hg orgánico, las alteraciones más importantes son las relacionadas con el neurodesarrollo, que ocasionan la disminución del coeficiente intelectual y retardo mental. Puede manifestarse con alteraciones visuales, hipoacusia, ataxia, temblor y alteraciones de la sensibilidad. En los últimos años, se ha asociado otra forma orgánica, el timerosal, con el autismo, pero todavía existen controversias al respecto. .,11-14 $^{8}$

Centrados en el concepto de desarrollo como origen de la salud y la enfermedad (Developmental Origins of Health and Disease, $\mathrm{DOHaD})$, se debe considerar que la exposición a metales pesados en las etapas tempranas de la vida conduce a la programación de enfermedades no transmisibles (ENT), que se manifestarán más tardíamente en la vida. ${ }^{12,14}$ La Agencia Internacional para la Investigación del Cáncer (International Agency for Research on Cancer, IARC) clasifica los compuestos orgánicos de $\mathrm{Hg}$ en el grupo 2B (probados efectos cancerígenos en animales de experimentación y posible en seres humanos). ${ }^{8}$ Los profesionales de la salud deben comprometerse y apoyar todas aquellas medidas destinadas a disminuir la exposición de los niños a los compuestos mercuriales.

\section{REFERENCIAS}

1. Ortez González C, Díaz Conradi A. Síndrome de GuillainBarré en la Infancia. An Pediatr Contin. 2013; 11(2):98-103.

2. Dreisbach R, Bev-Lorraine True. Tóxicos metálicos: Mercurio. En: Dreisbach R, Bev-Lorraine True. Manual de Toxicología Clínica de prevención, diagnóstico y tratamiento.

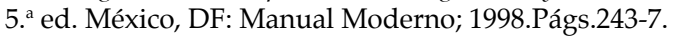

3. Caravati E, Erdman AR, Christianson G, Nelson LS, et al. Elemental mercury exposure an evidenced based consensus guideline for out- of hospital management. Clin Toxicol (Phila). 2008; 46(1):1-21.

4. Calello D, Henreting F. Lead. In: Hoffman RS, Howland MA, LewinNA, Nelson LS, etal.Golsfrank's Toxicologic Emergencies. 10th ed. New York: Mc Graw-Hill; 2015:Cap. 96.

5. Elberger S, Brody G. Cadmium, Mercury and Arsenic. In: Viccellio P. Emergency Toxicology. $5^{\mathrm{a}}$ ed. Philadelphia: Lippincott-Raven; 1998.Págs.381-4.

6. Greco V, Gastaldi P. D-Penicilamina. En Centro Nacional de Intoxicaciones. Guía de Antídotos y Tratamiento en Intoxicaciones. Buenos Aires: Hospital Nacional A. Posadas; 2019.Págs.73-4.

TABLA 4. Fuentes antropogénicas de mercurio

Relacionadas con la industria

Extracción de combustibles fósiles y metales preciosos.

Industrias cloroalcalinas y de la seda.

Pinturas, tinturas y cosméticos.

Fabricación de espejos, cementeras.

Centrales termoeléctricas, termostatos.

Lámparas fluorescentes y de bajo consumo, pilas.

Plaguicidas, biocidas.
Relacionadas con la salud

Instrumental médico (termómetros, esfingomanómetros, bujías).

Amalgamas dentales.

Productos de medicina ayurvédica.

Diuréticos y laxantes.

Antisépticos. 
7. Avaria MA, Kleinsteuber K, Castiglioni C. Enfermedades Neuromusculares en el recién nacido. En: Menéndez P, Hernández M, Pinto F. Neurología Perinatal. Santiago de Chile: Medigraphia; 2002.Págs.274-305.

8. Gaioli M, Amoedo D, Gonzalez D. Impacto del mercurio sobre la salud humana y el ambiente. Arch Argent Pediatr. 2012; 110(3):259-64.

9. Ortega García JA, Ferris Tortajada A, Cánovas Conesa A, García Castell J. Neurotóxicos medioambientales (y II). Metales: efectos adversos en el sistema nervioso fetal y posnatal. Acta Pediatr Esp. 2005; 63:182-92.

10. Organización Panamericana de la Salud. El mercurio y la salud. 2017. [Acceso: 15 de agosto de 2019]. Disponible en: http:/ / www.who.int/es/news-room/fact-sheets/detail/ mercury-and-health.
11. Crespo-López ME, Herculano AM, Corvelo TC, Do Nascimento JL. Mercurio y Neurotoxicidad. Rev Neurol. 2005; 40(7):441-7.

12. Rice KM, Walker EJJr, Wu M, Gillete C, et al. Environmental mercury and its toxic effects. J Prev Med Public Health. 2014; 47(2):74-83.

13. Ortega García JA, Ferris Tortajada J, López Andreu JA, Marco Macián A, et al. Hospitales Sostenibles(II). Mercurio: exposición pediátrica. Efectos adversos en la salud humana y medidas preventivas. Rev Esp Pediatr. 2003; 59(3):274-91.

14. CliftonJC2nd. Mercury exposure and publichealth. Pediatr Clin North Am. 2007; 54(2):237-69. 